\title{
Rotational Barrier and Bond Dissociation Energy and Enthalpy: Computational Study of the Substituent Effects in Para-Substituted Anilines and Phenols
}

\author{
Ali Hussain Yateem* \\ Department of Chemistry, College of Science, University of Bahrain, P. O. Box 32038, Sakhir, Kingdom of Bahrain
}

*Corresponding author:

email: alihali222@gmail.com

Received: August 26, 2021

Accepted: September 28, 2021

DOI: $10.22146 / \mathrm{ijc} .68687$

\begin{abstract}
This report presents the $\mathrm{N}-\mathrm{H}$ and $\mathrm{O}-\mathrm{H}$ bond dissociation energies (BDEs) and enthalpies (BDEts) of 27 para-substituted anilines and phenols using Density Functional Theory (DFT) with functional $\omega B 97 X-D$ and basis set $6-31 G^{* *}$. The computed BDEs/ BDEts show a strong correlation with the calculated rotational barrier (RB) around phenyl- $\mathrm{NH}_{2}$ and phenyl-OH bonds of the parent neutral molecules. Electron-withdrawing (EW) substituents increased $R B$ and BDEs/BDEts, while electron-donating (ED) substituents caused opposite behavior. Geometric, atomic, molecular, and spectroscopic properties of $\mathrm{NH}_{2}$ and $\mathrm{OH}$ groups in neutral anilinic and phenolic molecules exhibited excellent correlations with RB and BDEs/BDEts. The geometry around heteroatoms of the radicals displayed constant geometrical changes for all substituents. Spin density maps confirmed that the unpaired electrons in radicals are delocalized in heteroatoms and phenyl rings for all the para-substituents. Spin delocalization in both types of radicals was further enhanced in the presence of para-ED substituents. The increase in electronic density around heteroatoms of radicals with the strength of ED substituents was found proportional to that in neutral molecules. Therefore, the $\mathrm{N}-\mathrm{H}$ and $\mathrm{O}-\mathrm{H}$ BDE/BDEt are mainly governed by the stabilization/destabilization of the neutral molecules and, to a significantly lower extent, the stabilization of radicals in the case of strong ED groups.
\end{abstract}

Keywords: rotational barrier; bond dissociation energy; bond dissociation enthalpy; electron-donating/electron-withdrawing groups; substituent effect

\section{- INTRODUCTION}

Antioxidants such as phenolic compounds and aromatic amines protect free radical intermediates and reactive oxygen/nitrogen species in biological systems and synthetic products [1-9]. The radical scavenging mechanism is initiated by abstracting a hydrogen atom from the antioxidants [10-17]. The energy calculation for the breakage of a hydrogen bond is essential for understanding the antioxidant scavenging mechanism and is also fundamental for various chemical and biochemical transformations [10-11,18-20]. The bond dissociation energies (BDEs) and bond dissociation enthalpies (BDEts) are the most critical factors for the estimation of hydrogen atom abstraction and stability of the parent molecules as well as the radical products $[3,10,18,21-23]$.
BDEs and BDEts have been calculated experimentally and theoretically for para-substituted anilines and phenols with a very close agreement between the two approaches [2,24-31]. In almost all theoretical studies, the DFT functional has successfully reproduced the experimental BDEs [24-25,28,30]. It was concluded from the experimental and theoretical studies that EW groups in para position to phenols and anilines increase the $\mathrm{N}-\mathrm{H}$ and $\mathrm{O}-\mathrm{H}$ BDE/BDEt, while the opposite is true for ED groups [2,27-29,31]. Several studies also show good correlation with Hammett parameters [2,27-29,32]. Despite many studies on $\mathrm{BDE} / \mathrm{BDEt}$, the origin of the substituent effects on $\mathrm{BDE} / \mathrm{BDEt}$ in para-substituted anilines and phenols is not clearly elaborated.

Some studies suggest that para-electron- 
withdrawing (EW) groups in anilines and phenols stabilize the neutral molecules and destabilize the resulting radicals [28-29]. The converse holds good for electron-donating (ED) groups. Wu and Lai [33] proposed that stabilization of the phenoxyl radicals is more important than the neutral parent molecules in determining the $\mathrm{O}-\mathrm{H}$ BDEs. In their study, the stabilization of neutral para-substituted phenols in the presence of EW groups originates from the increase in $\pi$ delocalization throughout the whole molecule. The destabilization in the presence of ED groups occurs due to the repulsive $\pi$-saturation effect. $\mathrm{Wu}$ and Lai further suggested that spin delocalization stabilizes phenoxyl radicals in the case of both ED and EW substituents. Song et al. [32] indicated that the substituent effects on $\mathrm{N}-\mathrm{H}$ $\mathrm{BDE} / \mathrm{BDEt}$ are attributed mainly to the stabilization/destabilization of the neutral parasubstituted anilines and not to their corresponding radicals. The EW groups can interact with lone pair of electrons in $\mathrm{NH}_{2}$ more significantly than electrondonating groups due to the presence of low-lying unoccupied molecular orbitals in the formers. It was demonstrated by Zhang et al. [34] that stabilization of parent para-substituted phenols and the corresponding phenoxyl radicals is determined mainly by the resonance effect due to correlation of $\mathrm{O}-\mathrm{H}$ BDE with resonance parameter $\mathrm{R}^{+}$. The resonance effect causes a reduction of $\mathrm{O}-\mathrm{H}$ BDE in the case of ED groups and increases $\mathrm{O}-\mathrm{H}$ BDE with EW groups.

It is clear from the above discussion that BDEs/BDEts values depend on stabilization/ destabilization effects of substituent on both neutral parent molecules and their corresponding anilinyl and phenoxyl radicals. However, the origin of substituent effects is not yet fully clarified, especially for radicals. Some essential structural, atomic, molecular, and spectroscopic properties of neutral molecules and radicals have been neglected or considered incomprehensively in investigating the substituent effect. Moreover, BDEs and BDEts have been studied for typical substituents and correlated mainly to traditional Hammett parameters [2,27-29,32]. A clear distinction has not been made between the effect of substituent on neutral molecules and radicals. The current work bridges this literature gap to present BDEs/BDEts of 27 para-substituted anilines and phenols and their correlation to the rotational barrier (RB) around phenyl- $\mathrm{NH}_{2}$ and phenyl-OH bonds. Some geometric, atomic, and molecular parameters have been calculated in this study to investigate further the governing reason behind the trend of $\mathrm{BDE} / \mathrm{BDE}$ with the nature of the substituent. Previous studies have reported that $\mathrm{RB}$ is a valid and valuable measure for the stability in para-substituted anilines [35], parasubstituted benzaldehydes [36], as well as in disubstituted 1,3-butadienes [37] through resonance or inductive effects. Hence, the correlation of the BDEs/BDEts of 27 para-substituted anilines and phenols with $\mathrm{RB}$ is a particular focus of interest in the current work. The para position was selected since RBs were calculated before for para-substituted anilines and benzaldehydes [35-36]. Moreover, substituents in para position exhibit a more reliable trend in terms of the effect of substituents on BDE/BDEt compared to other positions $[24,26]$. Also, the available experimental data for N-H and O-H BDEs of para-substituted anilines and phenols are noticeably more than those of different positions [26-31].

\section{- COMPUTATIONAL METHODS}

All calculations were carried out using DFT level of theory with exchange-correlation functional of $\omega \mathrm{B} 97 \mathrm{X}$ $\mathrm{D}$ [38-39] and 6-31G** The computational calculations were executed using Spartan 14 (v. 1.1.4) [40]. The same method was used to calculate rotational barrier in parasubstituted anilines and benzaldehydes [35-36].

Bond dissociation energy, $\mathrm{BDE}$, was calculated according to Eq. (1)

$\mathrm{BDE}=\mathrm{E}_{\mathrm{o}}(\dot{\mathrm{R}})+\mathrm{E}_{\mathrm{o}}(\dot{\mathrm{H}})-\mathrm{E}_{\mathrm{o}}(\mathrm{R}-\mathrm{H})$

where $\mathrm{E}_{0}(\dot{\mathrm{R}}), \mathrm{E}_{\mathrm{o}}(\dot{\mathrm{H}})$, and $\mathrm{E}_{\mathrm{o}}(\mathrm{R}-\mathrm{H})$ are the total electronic energy of the radical, the hydrogen atom, and the neutral molecule, respectively.

Bond dissociation enthalpy, BDEt, was calculated using Eq. (2)

BDEt $=\mathrm{H}^{\circ}(\dot{\mathrm{R}})+\mathrm{H}^{\circ}(\dot{\mathrm{H}})-\mathrm{H}^{\circ}(\mathrm{R}-\mathrm{H})$

where $H^{\circ}(\dot{R}), H^{\circ}(\dot{H})$, and $H^{\circ}(R-H)$ are the total enthalpy of the radical, the hydrogen atom, and the neutral 
molecule, respectively. The electronic energies and enthalpies of all species were obtained directly from this software.

The internal rotation potential energy curves were obtained by performing geometry optimization calculations at a set of CCNH (for anilines) and $\mathrm{CCOH}$ (for phenols) dihedral angles ranging from 0 to $130^{\circ}$ with an increment of $10^{\circ}$. Close to the maximum state, the increment was decreased to $1^{\circ}$ and then to $0.1^{\circ}$. The minimum equilibrium states were optimized separately. The internal $\mathrm{RB}$ around the phenyl- $\mathrm{NH}_{2}$ and phenyl-OH single bonds was considered between the minimum (equilibrium) and maximum conditions. The minimum states appear at dihedral angles between $18.50^{\circ}$ and $28.38^{\circ}$ for anilinyl compounds and between $-0.87^{\circ}$ and $0.43^{\circ}$ for phenolic compounds. The maximum conditions appear at dihedral angles between $120.50^{\circ}$ and $122.10^{\circ}$ for anilinyl compounds and between $90.70^{\circ}$ and $92.40^{\circ}$ for phenolic compounds. Energies of the minimum and maximum states were corrected for zero-point energies, and transition states were confirmed using frequency analysis.

The minimum ionization potential was calculated on the lone pair of the amino nitrogen region in the equilibrium states of neutral anilinyl molecules and their corresponding radicals. The maximum (positive) electrostatic potential was calculated if it occupied amino hydrogens in the equilibrium states of neutral anilinyl molecules and their corresponding radicals. The minimum (negative) electrostatic potential was calculated using hydroxyl oxygen in equilibrium states of neutral phenolic molecules and their corresponding radicals.

Substituents considered here are all neutral, and they are ranged from weakly to strongly electronwithdrawing/donating substituents. The ED substituents include amino, alkoxy, and alkyl groups. The EW substituents include nitro, cyano, carbonyl, and sulfonyl groups and groups such as $\mathrm{SiF}_{3}$ and $\mathrm{CF}_{3}$. Other substituents like halides are considered borderline groups. In the case of more than one conformer for a molecule, the conformer with the lowest energy was chosen after performing conformers distribution analysis.

\section{- RESULTS AND DISCUSSION}

\section{Para-Substituted Anilines}

Table 1 shows calculated $\mathrm{RB}$ around phenyl- $\mathrm{NH}_{2}$ bond, $\mathrm{RB}_{\mathrm{C}-\mathrm{N}}$, calculated $\mathrm{N}-\mathrm{H} \mathrm{BDE}$ and $\mathrm{BDEt}$, and experimental BDE $[28,30]$ of 27 para-substituted anilines. The substituents are arranged in terms of decreasing values of $\mathrm{RBs}_{\mathrm{C}-\mathrm{N}}$. It can be seen from Table 1 that calculated BDEts are well consistent with the experimental values. An observation of the table further shows that with the increase in $\mathrm{RB}_{\mathrm{C}-\mathrm{N}}$, the values of both $\mathrm{N}-\mathrm{H}$ BDE and BDEt increase, and the EW character of para-substituent gets enhanced. It has been established previously that EW groups in the para position of aniline increase the $\mathrm{N}-\mathrm{H} \mathrm{BDE} / \mathrm{BDEt}$, and the opposite is true for ED groups [28-29,31]. It has also been shown previously that $\mathrm{RBs}_{\mathrm{C}-\mathrm{N}}$ increases with the strength of EW properties of para-substituents in anilines [35]. Fig. 1 shows that $\mathrm{N}-\mathrm{H} \mathrm{BDE} / \mathrm{BDE}$ correlates well with the $\mathrm{RB}_{\mathrm{C}-}$ ${ }_{\mathrm{N}}$ around phenyl- $\mathrm{NH}_{2}$ bond. Since BDEs are strongly correlated with BDEts $\left(\mathrm{R}^{2}=0.999\right)$, the data for BDEt were used in most of the graphs presented in this work.

Table 2 shows phenyl- $\mathrm{NH}_{2}$ bond length, $R(\mathrm{C}-\mathrm{N})$, and phenyl- $\mathrm{N}-\mathrm{H}$ bond angle, $A(\mathrm{C}-\mathrm{N}-\mathrm{H})$, of parasubstituted anilines and their corresponding radicals. The decrease in $\mathrm{C}-\mathrm{N}$ bond length and the increase of $\mathrm{C}-$ $\mathrm{N}-\mathrm{H}$ bond angle of neutral molecules from the bottom to the top of the table are the outcomes of increased $\pi$ delocalization corresponding to the strength of EW

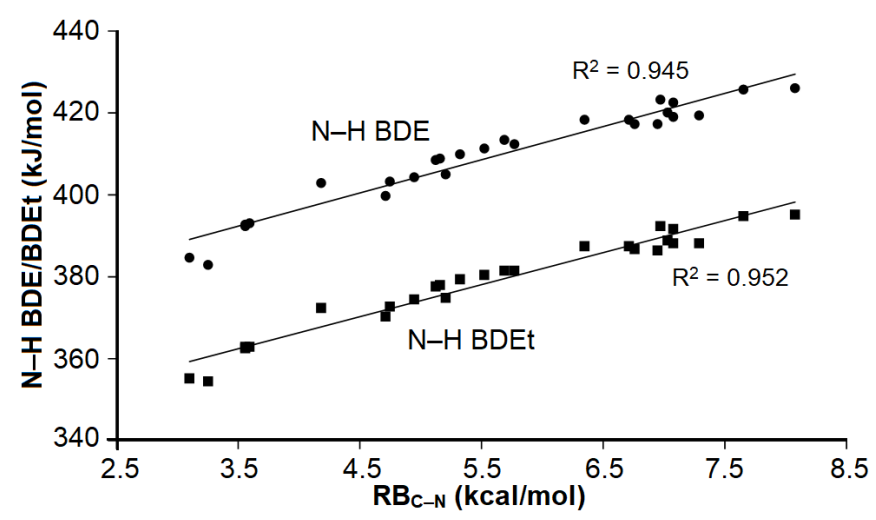

Fig 1. $\mathrm{N}-\mathrm{H}$ bond dissociation energy (BDE) and enthalpy (BDEt) vs. rotational barrier around the phenyl- $\mathrm{NH}_{2}$ bond of para-substituted anilines 
Table 1. The calculated rotational barrier around phenyl$\mathrm{NH}_{2}$ bond $\left(\mathrm{RB}_{\mathrm{C}-\mathrm{N}}\right)$, calculated bond dissociation energy (BDE) and enthalpy (BDEt), and experimental BDE of para-substituted anilines

\begin{tabular}{|c|c|c|c|c|}
\hline Substituent & $\begin{array}{c}\mathrm{RB}_{\mathrm{C}-\mathrm{N}} \\
(\mathrm{kcal} / \mathrm{mol})\end{array}$ & $\begin{array}{c}\text { BDE } \\
(\mathrm{kJ} / \mathrm{mol})\end{array}$ & $\begin{array}{c}\text { BDEt } \\
(\mathrm{kJ} / \mathrm{mol})\end{array}$ & $\begin{array}{l}\text { Expt BDE }{ }^{a} \\
(\mathrm{~kJ} / \mathrm{mol})\end{array}$ \\
\hline $\mathrm{COCl}$ & 8.08 & 426.09 & 395.09 & \\
\hline $\mathrm{NO}_{2}$ & 7.65 & 425.68 & 394.91 & 405 \\
\hline $\mathrm{CHO}$ & 7.29 & 419.30 & 388.31 & \\
\hline $\mathrm{COOH}$ & 7.08 & 418.95 & 388.09 & \\
\hline $\mathrm{SiF}_{3}$ & 7.08 & 422.49 & 391.83 & \\
\hline $\mathrm{CN}$ & 7.03 & 420.00 & 388.97 & 398,384 \\
\hline $\mathrm{SO}_{2} \mathrm{CH}_{3}$ & 6.97 & 423.17 & 392.33 & \\
\hline $\mathrm{COCH}_{3}$ & 6.94 & 417.25 & 386.28 & 394,379 \\
\hline $\mathrm{CO}_{2} \mathrm{CH}_{3}$ & 6.76 & 417.30 & 386.82 & \\
\hline SCN & 6.71 & 418.28 & 387.50 & \\
\hline $\mathrm{CF}_{3}$ & 6.34 & 418.30 & 387.55 & 404,385 \\
\hline $\mathrm{SCH}_{3}$ & 5.77 & 412.36 & 381.37 & \\
\hline $\mathrm{SH}$ & 5.69 & 413.47 & 381.62 & \\
\hline I & 5.52 & 411.36 & 380.58 & 373 \\
\hline $\mathrm{Br}$ & 5.32 & 409.96 & 379.31 & 386 \\
\hline Phenyl & 5.21 & 405.17 & 374.76 & \\
\hline $\mathrm{Cl}$ & 5.16 & 408.80 & 378.13 & 387 \\
\hline $\mathrm{H}$ & 5.12 & 408.36 & 377.63 & $386,373,375$ \\
\hline $\mathrm{C}\left(\mathrm{CH}_{3}\right)_{3}$ & 4.95 & 404.17 & 374.56 & 372 \\
\hline $\mathrm{CH}_{3}$ & 4.75 & 403.11 & 372.59 & $385,371,366$ \\
\hline OPhenyl & 4.71 & 399.90 & 370.15 & \\
\hline $\mathrm{F}$ & 4.18 & 402.92 & 372.41 & 372 \\
\hline $\mathrm{OCH}_{3}$ & 3.59 & 393.12 & 362.94 & 378,365 \\
\hline $\mathrm{OH}$ & 3.56 & 392.56 & 362.83 & \\
\hline $\mathrm{OCH}_{2} \mathrm{CH}_{3}$ & 3.56 & 392.66 & 362.51 & \\
\hline $\mathrm{N}\left(\mathrm{CH}_{3}\right)_{2}$ & 3.25 & 382.84 & 354.63 & \\
\hline $\mathrm{NH}_{2}$ & 3.10 & 384.80 & 355.30 & 360 \\
\hline
\end{tabular}

${ }^{\text {a }}$ from ref. [28, 30]

groups [35]. The increase of double bond character of C$\mathrm{N}$ bond and the decrease of pyramidization of $\mathrm{NH}_{2}$ group reflect the involvement of lone pair of amino nitrogen in $\pi$-electron resonance stabilization [35]. Fig. 2 and $S 1$ show that $\mathrm{C}-\mathrm{N}$ bond lengths and $\mathrm{C}-\mathrm{N}-\mathrm{H}$ bond angles strongly correlate with $\mathrm{RB}_{\mathrm{C}-\mathrm{N}}$. On the other hand, the phenyl- $\mathrm{N} H$ bond distances and phenyl- $-\dot{N}-\mathrm{H}$ bond angles of the anilinyl radicals are almost constant at $1.332 \AA$ and $109.10^{\circ}$, respectively, for para substituents. The $\mathrm{N}-\mathrm{H}$ bond distance is also stable at $1.024 \AA$ for all substituents versus
Table 2. Phenyl- $\mathrm{NH}_{2}$ bond length, $\mathrm{R}(\mathrm{C}-\mathrm{N})$, phenyl-N$\mathrm{H}$ bond angle, $\mathrm{A}(\mathrm{C}-\mathrm{N}-\mathrm{H})$, para-substituted anilines, and corresponding aniline radicals

\begin{tabular}{|c|c|c|c|c|}
\hline \multirow[b]{2}{*}{ Substituent } & \multicolumn{2}{|c|}{ Anilines } & \multicolumn{2}{|c|}{ Anilinyls } \\
\hline & $\begin{array}{c}\mathrm{R}(\mathrm{C}-\mathrm{N}) \\
(\AA)\end{array}$ & $\begin{array}{c}\mathrm{A}(\mathrm{C}-\mathrm{N}-\mathrm{H}) \\
(\mathrm{deg})\end{array}$ & $\begin{array}{c}\mathrm{R}(\mathrm{C}-\dot{\mathrm{N}}) \\
(\AA)\end{array}$ & $\begin{array}{c}\mathrm{A}(\mathrm{C}-\mathrm{N}-\mathrm{H}) \\
(\mathrm{deg})\end{array}$ \\
\hline $\mathrm{COCl}$ & 1.373 & 117.97 & 1.330 & 109.34 \\
\hline $\mathrm{NO}_{2}$ & 1.375 & 117.64 & 1.332 & 109.38 \\
\hline $\mathrm{CHO}$ & 1.379 & 117.05 & 1.329 & 109.30 \\
\hline $\mathrm{COOH}$ & 1.381 & 116.72 & 1.332 & 109.16 \\
\hline $\mathrm{SiF}_{3}$ & 1.380 & 116.84 & 1.334 & 109.16 \\
\hline $\mathrm{CN}$ & 1.379 & 117.02 & 1.330 & 109.36 \\
\hline $\mathrm{SO}_{2} \mathrm{CH}_{3}$ & 1.379 & 117.22 & 1.335 & 109.23 \\
\hline $\mathrm{COCH}_{3}$ & 1.382 & 116.60 & 1.330 & 109.20 \\
\hline $\mathrm{CO}_{2} \mathrm{CH}_{3}$ & 1.383 & 116.42 & 1.332 & 109.12 \\
\hline SCN & 1.380 & 116.97 & 1.331 & 109.25 \\
\hline $\mathrm{CF}_{3}$ & 1.385 & 116.18 & 1.335 & 109.12 \\
\hline $\mathrm{SCH}_{3}$ & 1.389 & 115.58 & 1.334 & 109.06 \\
\hline $\mathrm{SH}$ & 1.388 & 115.66 & 1.334 & 109.07 \\
\hline I & 1.389 & 115.56 & 1.333 & 109.16 \\
\hline $\mathrm{Br}$ & 1.390 & 115.42 & 1.332 & 109.15 \\
\hline Phenyl & 1.392 & 115.10 & 1.33 & 109.00 \\
\hline $\mathrm{Cl}$ & 1.391 & 115.30 & 1.332 & 109.15 \\
\hline $\mathrm{H}$ & 1.394 & 114.91 & 1.336 & 108.94 \\
\hline $\mathrm{C}\left(\mathrm{CH}_{3}\right)_{3}$ & 1.396 & 114.61 & 1.334 & 108.86 \\
\hline $\mathrm{CH}_{3}$ & 1.397 & 114.54 & 1.333 & 108.92 \\
\hline OPhenyl & 1.394 & 114.99 & 1.334 & 109.06 \\
\hline $\mathrm{F}$ & 1.397 & 114.51 & 1.334 & 109.07 \\
\hline $\mathrm{OCH}_{3}$ & 1.402 & 113.87 & 1.332 & 108.96 \\
\hline $\mathrm{OH}$ & 1.403 & 113.82 & 1.332 & 108.98 \\
\hline $\mathrm{OCH}_{2} \mathrm{CH}_{3}$ & 1.402 & 113.80 & 1.332 & 108.94 \\
\hline $\mathrm{N}\left(\mathrm{CH}_{3}\right)_{2}$ & 1.404 & 113.55 & 1.328 & 108.83 \\
\hline $\mathrm{NH}_{2}$ & 1.405 & 113.60 & 1.329 & 108.92 \\
\hline
\end{tabular}

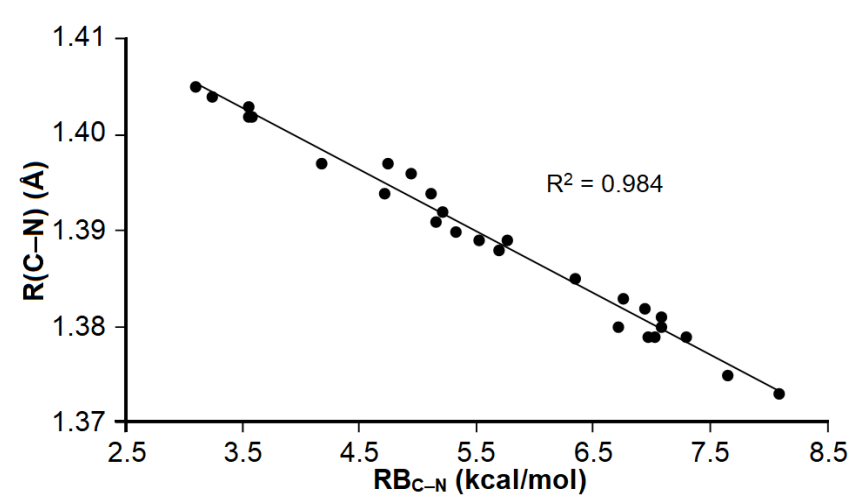

Fig 2. Phenyl- $\mathrm{NH}_{2}$ bond distance vs. rotational barrier around phenyl- $-\mathrm{NH}_{2}$ bond in para-substituted anilines 
a slight decrease with $\mathrm{RB}$ in neutral molecules from 1.010-1.006 $\AA$. The C-N and $\dot{\mathrm{N}}-\mathrm{H}$ bond distances and C$\dot{\mathrm{N}}-\mathrm{H}$ bond angles in anilinyl radicals are close to those calculated for 2-propanimine (1.273 $\AA, 1.023 \AA$, and $110.14^{\circ}$, respectively) using the $\omega \mathrm{B} 97 \mathrm{X}-\mathrm{D} / 6-31 \mathrm{G}^{\star *}$.

The spin density map representing unpaired electron location [41-42] is shown for unsubstituted and para- $N, N$-dimethylamino anilinyl radicals in Fig. 3 . The blue color on the spin density map indicates a high spin density area, and the electron density decreases from blue to red. It can be seen that the unpaired electron (blue color) is located mainly in the vicinity of $\dot{N}$ atom and the ortho and para positions. The spin density is more intense on $\mathrm{N}$ atom than the phenyl positions, especially in the case of strong donor $\mathrm{N}\left(\mathrm{CH}_{3}\right)_{2}$ at the para position. The fraction of spin density on $\mathrm{N}$ was found to increase with the strength of the ED group. The spin density maps of anilinyl radicals with para-EW groups and borderline substituents are similar to that of unsubstituted anilinyl radicals.

Table $\mathrm{S} 2$ shows the $\mathrm{NH}_{2}$ symmetric and asymmetric stretching frequencies, $v_{\mathrm{s}}\left(\mathrm{NH}_{2}\right)$ and $v_{\mathrm{a}}\left(\mathrm{NH}_{2}\right)$, respectively, for neutral anilinic compounds [35] and the $\dot{\mathrm{N} H}$ stretching frequency, $v(\dot{\mathrm{N}} \mathrm{H})$, of the corresponding anilinyl radicals. It is observable from the table that both $v_{\mathrm{s}}\left(\mathrm{NH}_{2}\right)$ and $v_{\mathrm{a}}\left(\mathrm{NH}_{2}\right)$ increase with the strength of EW character of the para-substituent and hence with $\mathrm{RB}_{\mathrm{C}-\mathrm{N}}$, the $v(\dot{\mathrm{NH}})$ is roughly constant at an average value of $3489 \mathrm{~cm}^{-1}$. Fig. S2 shows that $v_{s}\left(\mathrm{NH}_{2}\right)$ and $v_{a}\left(\mathrm{NH}_{2}\right)$ are linearly proportional to $\mathrm{RB}_{\mathrm{C}-\mathrm{N}}$.

Table 3 shows calculated minimum ionization potential around amino $\mathrm{N}$ atom, $\mathrm{IP}_{\min }$, maximum (positive) electrostatic potential around the amino $\mathrm{H}$ atom, $\mathrm{ES}_{\max }$, and the natural partial charge on amino $\mathrm{N}$ atom, $\mathrm{Q}_{\mathrm{n}}(\mathrm{N})$, of neutral and corresponding radicals of para-substituted anilines. Tabulated data for both neutral molecules and radicals reveal that as the strength of the EW substituent increases, minimum IP increases, $\mathrm{ES}_{\max }$ increases, and $\mathrm{Q}_{\mathrm{n}}(\mathrm{N})$ becomes less negative. However, the opposite trend is actual for ED substituents. For neutral molecules, strengthening the electron-acceptor para-substituent leads to a decrease in electronic density

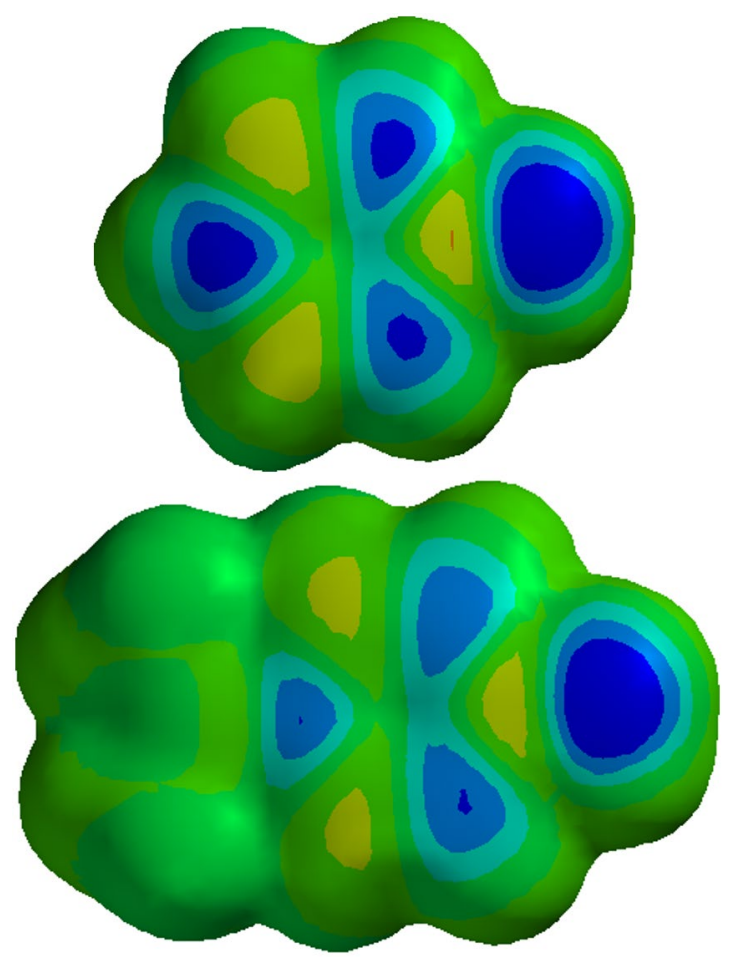

Fig 3. Spin density map for unsubstituted anilinyl (top) and para- $N, N$-dimethylamino anilinyl (bottom) radicals

Table 3. Minimum ionization potential around amino $\mathrm{N}$ atom, $\mathrm{IP}_{\min }$, maximum (positive) electrostatic potential around the amino $\mathrm{H}$ atom, $\mathrm{ES}_{\max }$, and the natural partial charge on amino $\mathrm{N}$ atom, $\mathrm{Q}_{\mathrm{n}}(\mathrm{N})$, of para-substituted anilines and the corresponding anilinyl radicals

\begin{tabular}{lcccccc}
\hline \multirow{3}{*}{ Substituent Anilines } & \multicolumn{3}{c}{ Anilinyls } \\
\cline { 2 - 7 } & $\begin{array}{c}\mathrm{IP}_{\min } \\
(\mathrm{eV})\end{array}$ & $\begin{array}{c}\mathrm{ES}_{\max } \\
(\mathrm{kJ} / \mathrm{mol})\end{array}$ & $\begin{array}{c}\mathrm{Q}_{\mathrm{n}}(\mathrm{N}) \\
(\mathrm{e})\end{array}$ & $\begin{array}{c}\mathrm{IP}_{\min } \\
(\mathrm{eV})\end{array}$ & $\begin{array}{c}\mathrm{ES}_{\max } \\
(\mathrm{kJ} / \mathrm{mol})\end{array}$ & $\begin{array}{c}\mathrm{Q}_{\mathrm{n}}(\mathrm{N}) \\
(\mathrm{e})\end{array}$ \\
\hline $\mathrm{COCl}$ & 11.41 & 247.48 & -0.846 & 11.09 & 185.12 & -0.522 \\
$\mathrm{NO}_{2}$ & 11.39 & 248.19 & -0.848 & 11.16 & 196.11 & -0.520 \\
$\mathrm{CHO}$ & 11.07 & 227.97 & -0.852 & 10.88 & 167.80 & -0.534 \\
$\mathrm{COOH}$ & 10.94 & & -0.855 & 10.76 & & -0.534 \\
$\mathrm{SiF}_{3}$ & 11.11 & 228.98 & -0.854 & 10.94 & & -0.527 \\
$\mathrm{CN}$ & 11.22 & 239.89 & -0.853 & 11.03 & 187.05 & -0.531 \\
\hline
\end{tabular}


Table 3. Minimum ionization potential around amino $\mathrm{N}$ atom, $\mathrm{IP}_{\min }$, maximum (positive) electrostatic potential around the amino $\mathrm{H}$ atom, $\mathrm{ES}_{\max }$, and the natural partial charge on amino $\mathrm{N}$ atom, $\mathrm{Q}_{\mathrm{n}}(\mathrm{N})$, of para-substituted anilines and the corresponding anilinyl radicals (Continued)

\begin{tabular}{|c|c|c|c|c|c|c|}
\hline \multirow[b]{2}{*}{ Substituent } & \multicolumn{3}{|c|}{ Anilines } & \multicolumn{3}{|c|}{ Anilinyls } \\
\hline & $\begin{array}{l}I_{\text {min }} \\
(\mathrm{eV})\end{array}$ & $\begin{array}{c}\mathrm{ES}_{\max } \\
(\mathrm{kJ} / \mathrm{mol})\end{array}$ & $\begin{array}{c}\mathrm{Q}_{\mathrm{n}}(\mathrm{N}) \\
(\mathrm{e})\end{array}$ & $\begin{array}{l}\mathrm{IP}_{\text {min }} \\
(\mathrm{eV})\end{array}$ & $\begin{array}{c}\mathrm{ES}_{\max } \\
(\mathrm{kJ} / \mathrm{mol})\end{array}$ & $\begin{array}{c}\mathrm{Q}_{\mathrm{n}}(\dot{\mathrm{N}}) \\
(\mathrm{e})\end{array}$ \\
\hline $\mathrm{SO}_{2} \mathrm{CH}_{3}$ & 11.20 & 237.49 & -0.853 & 11.00 & 184.64 & -0.524 \\
\hline $\mathrm{COCH}_{3}$ & 10.91 & 216.62 & -0.855 & 10.75 & 158.30 & -0.537 \\
\hline $\mathrm{CO}_{2} \mathrm{CH}_{3}$ & 10.84 & 210.43 & -0.856 & 10.71 & 152.94 & -0.537 \\
\hline SCN & & 235.57 & -0.854 & & 169.57 & -0.544 \\
\hline $\mathrm{CF}_{3}$ & 10.92 & 219.22 & -0.858 & 10.84 & 170.96 & -0.532 \\
\hline $\mathrm{SCH}_{3}$ & & 203.00 & -0.862 & & 151.03 & -0.544 \\
\hline SH & & 206.54 & -0.862 & & 157.74 & -0.541 \\
\hline I & & 210.75 & -0.862 & & 159.99 & -0.545 \\
\hline $\mathrm{Br}$ & 10.73 & 210.73 & -0.863 & 10.69 & 161.18 & -0.547 \\
\hline Phenyl & 10.48 & 192.83 & -0.865 & 10.40 & 136.38 & -0.559 \\
\hline $\mathrm{Cl}$ & 10.70 & 209.12 & -0.864 & 10.66 & 160.63 & -0.548 \\
\hline $\mathrm{H}$ & 10.39 & 184.66 & -0.867 & 10.45 & 134.43 & -0.548 \\
\hline $\mathrm{C}\left(\mathrm{CH}_{3}\right)_{3}$ & 10.32 & 182.06 & -0.868 & 10.31 & 125.71 & -0.558 \\
\hline $\mathrm{CH}_{3}$ & 10.30 & 181.73 & -0.869 & 10.30 & 130.54 & -0.559 \\
\hline OPhenyl & 10.54 & 199.61 & -0.866 & 10.53 & 143.17 & -0.553 \\
\hline $\mathrm{F}$ & 10.46 & 193.89 & -0.869 & 10.54 & 149.28 & -0.556 \\
\hline $\mathrm{OCH}_{3}$ & 10.17 & 177.42 & -0.872 & 10.16 & 120.34 & -0.578 \\
\hline $\mathrm{OH}$ & 10.20 & & -0.873 & 10.22 & & -0.577 \\
\hline $\mathrm{OCH}_{2} \mathrm{CH}_{3}$ & 10.16 & 172.64 & -0.873 & 10.14 & 114.96 & -0.579 \\
\hline $\mathrm{N}\left(\mathrm{CH}_{3}\right)_{2}$ & 10.01 & & -0.874 & 9.79 & & -0.604 \\
\hline $\mathrm{NH}_{2}$ & 10.04 & 167.84 & -0.874 & 9.93 & & -0.597 \\
\hline
\end{tabular}

around the amino group as the lone pair on nitrogen atom becomes more involved in $\pi$-delocalization that extends throughout the whole molecules $[33,35]$. This phenomenon implies more energy required to remove lone pair electrons on the nitrogen atom, an increase in acidity of amino hydrogen, and a less negative charge on amino $\mathrm{N}$ [35]. While for radicals, the data clearly show an increase in electron density around $\dot{\mathrm{NH}}$ moiety with increasing ED ability of the para-substituent. However, the change in electronic density around the radical $\mathrm{NH}$ group is almost parallel to that around the amino group in neutral molecules, as shown in Fig. 4, S3, and S4. Therefore, the effect of substituents on radicals correlates with neutral molecules in terms of a shift in electronic density.

\section{Para-Substituted Phenols}

Table 4 shows calculated rotational barrier around phenyl-

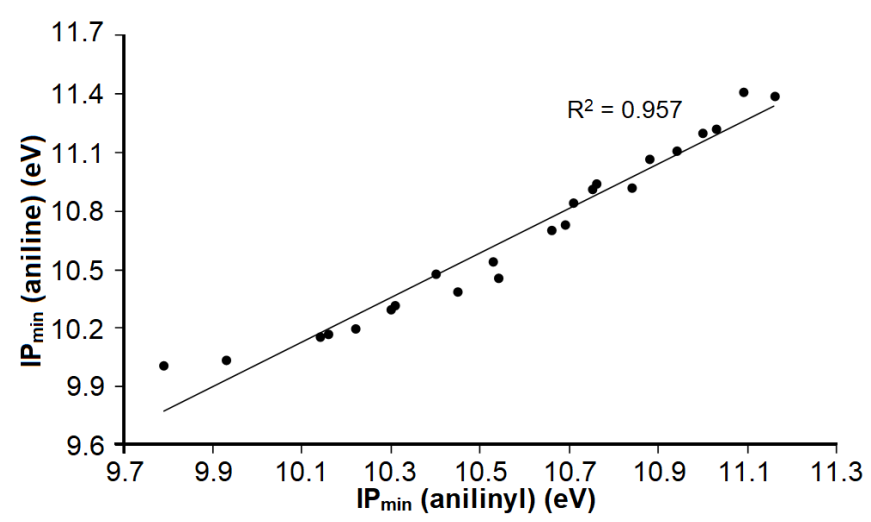

Fig 4. The minimum ionization potential of neutral para-substituted anilines vs. minimum ionization potential of the corresponding anilinyl radicals

$\mathrm{OH}$ bond, $\mathrm{RB}_{\mathrm{C}-\mathrm{O}}$, calculated $\mathrm{O}-\mathrm{H} \mathrm{BDE}$ and BDEt, and experimental BDE [12,16] for all selected 27 parasubstituted phenols. Unfortunately, the experimental BDEs fall in a rather broad range for most substituents, 
Table 4. The calculated rotational barrier around phenyl-OH bond $\left(\mathrm{RB}_{\mathrm{C}-\mathrm{O}}\right)$, calculated bond dissociation energy (BDE) and enthalpy (BDEt), and experimental BDE of para-substituted phenols

\begin{tabular}{|c|c|c|c|c|c|}
\hline Substituent & $\begin{array}{c}\mathrm{RB}_{\mathrm{C}-\mathrm{O}} \\
(\mathrm{kcal} / \mathrm{mol})\end{array}$ & $\begin{array}{c}\text { BDE } \\
(\mathrm{kJ} / \mathrm{mol})\end{array}$ & $\begin{array}{c}\text { BDEt } \\
(\mathrm{kJ} / \mathrm{mol})\end{array}$ & $\begin{array}{c}\text { Expt BDE }^{\mathrm{a}} \\
(\mathrm{kJ} / \mathrm{mol})\end{array}$ & $\begin{array}{c}\text { Expt BDE }^{\mathrm{b}} \\
(\mathrm{kJ} / \mathrm{mol})\end{array}$ \\
\hline $\mathrm{COCl}$ & 4.38 & 403.40 & 372.48 & & \\
\hline $\mathrm{NO}_{2}$ & 4.15 & 403.94 & 373.32 & $394-399$ & 373-399 \\
\hline $\mathrm{CHO}$ & 4.13 & 395.50 & 364.96 & & \\
\hline $\mathrm{SO}_{2} \mathrm{CH}_{3}$ & 4.10 & 402.25 & 371.20 & 397 & \\
\hline $\mathrm{SiF}_{3}$ & 3.98 & 401.09 & 370.75 & & \\
\hline $\mathrm{COOH}$ & 3.95 & 395.74 & 364.64 & & 372 \\
\hline $\mathrm{CO}_{2} \mathrm{CH}_{3}$ & 3.92 & 393.74 & 363.72 & & \\
\hline $\mathrm{CN}$ & 3.88 & 396.27 & 365.48 & $388-397$ & $370-397$ \\
\hline SCN & 3.87 & 401.13 & 370.09 & & \\
\hline $\mathrm{COCH}_{3}$ & 3.83 & 392.66 & 362.16 & $370-391$ & $366-391$ \\
\hline $\mathrm{CF}_{3}$ & 3.66 & 396.57 & 366.02 & 398,401 & $382-401$ \\
\hline $\mathrm{SCH}_{3}$ & 3.57 & 388.49 & 358.12 & & \\
\hline SH & 3.49 & 390.13 & 358.84 & & \\
\hline I & 3.23 & 386.49 & 356.34 & & \\
\hline $\mathrm{H}$ & 3.19 & 385.44 & 355.23 & $367-379$ & 368,369 \\
\hline Phenyl & 3.19 & 378.34 & 349.43 & 366 & $355-369$ \\
\hline $\mathrm{C}\left(\mathrm{CH}_{3}\right)_{3}$ & 3.18 & 382.20 & 352.55 & $357-374$ & $357-374$ \\
\hline $\mathrm{Br}$ & 3.14 & 384.82 & 354.93 & $368-382$ & $356-382$ \\
\hline $\mathrm{Cl}$ & 3.06 & 383.47 & 353.56 & $366-381$ & $267-391$ \\
\hline Ophenyl & 2.89 & 369.01 & 340.16 & & \\
\hline $\mathrm{CH}_{3}$ & 2.88 & 377.71 & 347.68 & $360-374$ & $360-374$ \\
\hline $\mathrm{F}$ & 2.59 & 376.89 & 347.37 & 365 & $354-382$ \\
\hline $\mathrm{OH}$ & 2.28 & 362.96 & 334.44 & 335,341 & $336-354$ \\
\hline $\mathrm{OCH}_{2} \mathrm{CH}_{3}$ & 2.26 & 362.26 & 333.33 & & \\
\hline $\mathrm{OCH}_{3}$ & 2.26 & 362.99 & 334.14 & $341-356$ & $346-361$ \\
\hline $\mathrm{NH}_{2}$ & 2.12 & 350.35 & 321.87 & $316-355$ & $316-357$ \\
\hline $\mathrm{N}\left(\mathrm{CH}_{3}\right)_{2}$ & 2.06 & 346.77 & 320.20 & $310-338$ & $310-336$ \\
\hline
\end{tabular}

so a direct comparison with calculated BDE/BDEt is troublesome. Still, most of the calculated O-H BDE and BDEt are within the range of experimental data.

The data of para-substituted anilines listed in Table 4 show that as $\mathrm{RB}_{\mathrm{C}-\mathrm{O}}$ increases, the values of both $\mathrm{O}-\mathrm{H}$ $\mathrm{BDE}$ and BDEt increase, and this increase in $\mathrm{RB}_{\mathrm{C}-\mathrm{O}}$ and $\mathrm{BDE} / \mathrm{BDE}$ is proportional to the strength of EW parasubstituent. Fig. 5 demonstrates a strong correlation between $\mathrm{RB}_{\mathrm{C}-\mathrm{O}}$ and $\mathrm{O}-\mathrm{H}$ BDE/BDEt.

Table 5 shows calculated $\mathrm{RB}_{\mathrm{C}-\mathrm{O}}$, phenyl-OH bond length, $\mathrm{R}(\mathrm{C}-\mathrm{O})$, a natural partial charge on $\mathrm{O}$ of hydroxyl group, $\mathrm{Q}_{\mathrm{n}}(\mathrm{O})$, and minimum (negative) electrostatic potential in the vicinity of lone pair of hydroxyl $\mathrm{O}, \mathrm{ES}_{\min }$,

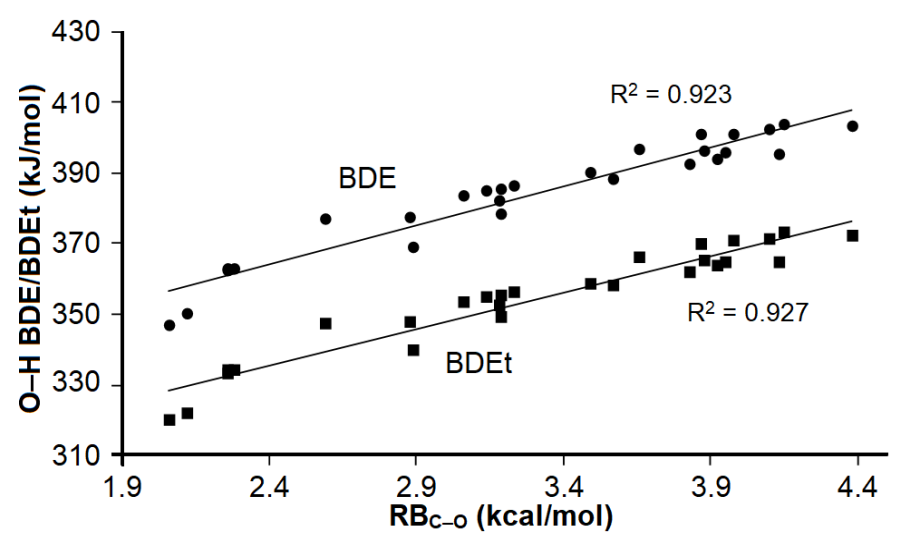

Fig 5. $\mathrm{O}-\mathrm{H}$ bond dissociation energy (BDE) and enthalpy (BDEt) vs. rotational barrier around the phenyl-OH bond of para-substituted phenols 
Table 5. Phenyl-OH bond length, $\mathrm{R}(\mathrm{C}-\mathrm{O}$, minimum (negative) electrostatic potential around lone pair of hydroxyl $\mathrm{O}, \mathrm{ES}_{\min }$, and natural partial charge on hydroxyl $\mathrm{O}$ atom, $\mathrm{Q}_{\mathrm{n}}(\mathrm{O})$, of para-substituted phenols and the corresponding radicals

\begin{tabular}{|c|c|c|c|c|c|c|}
\hline \multirow[b]{2}{*}{ Substituent } & \multicolumn{3}{|c|}{ Phenols } & \multicolumn{3}{|c|}{ Phenoxyls } \\
\hline & $\begin{array}{c}\mathrm{R}(\mathrm{C}-\mathrm{O}) \\
(\AA)\end{array}$ & $\begin{array}{c}\mathrm{ES}_{\min } \\
(\mathrm{kJ} / \mathrm{mol})\end{array}$ & $\begin{array}{c}\mathrm{Q}_{\mathrm{n}}(\mathrm{O}) \\
(\mathrm{e})\end{array}$ & $\begin{array}{c}\mathrm{R}(\mathrm{C}-\dot{\mathrm{O}}) \\
(\AA)\end{array}$ & $\begin{array}{c}\mathrm{ES}_{\min } \\
(\mathrm{kJ} / \mathrm{mol})\end{array}$ & $\begin{array}{c}\mathrm{Q}_{\mathrm{n}}(\dot{\mathrm{O}}) \\
(\mathrm{e})\end{array}$ \\
\hline $\mathrm{COCl}$ & 1.349 & & -0.688 & 1.244 & & -0.463 \\
\hline $\mathrm{NO}_{2}$ & 1.350 & & -0.689 & 1.245 & & -0.458 \\
\hline $\mathrm{CHO}$ & 1.352 & & -0.693 & 1.244 & & -0.475 \\
\hline $\mathrm{SO}_{2} \mathrm{CH}_{3}$ & 1.352 & & -0.692 & 1.248 & & -0.463 \\
\hline $\mathrm{SiF}_{3}$ & 1.352 & & -0.693 & 1.248 & & -0.466 \\
\hline $\mathrm{COOH}$ & 1.353 & & -0.694 & 1.246 & & -0.474 \\
\hline $\mathrm{CO}_{2} \mathrm{CH}_{3}$ & 1.354 & & -0.695 & 1.246 & & -0.478 \\
\hline $\mathrm{CN}$ & 1.352 & & -0.691 & 1.244 & & -0.471 \\
\hline SCN & 1.352 & & -0.692 & 1.247 & & \\
\hline $\mathrm{COCH}_{3}$ & 1.354 & & -0.694 & 1.245 & & -0.479 \\
\hline $\mathrm{CF}_{3}$ & 1.354 & -104.65 & -0.696 & 1.248 & -157.27 & -0.470 \\
\hline $\mathrm{SCH}_{3}$ & 1.357 & & -0.699 & 1.247 & & -0.484 \\
\hline $\mathrm{SH}$ & 1.357 & -116.54 & -0.699 & 1.247 & -169.42 & -0.480 \\
\hline I & 1.356 & -110.87 & -0.698 & 1.246 & -169.14 & -0.485 \\
\hline $\mathrm{H}$ & 1.360 & -134.26 & -0.703 & 1.249 & -183.05 & -0.486 \\
\hline Phenyl & 1.359 & -128.06 & -0.702 & 1.246 & -191.51 & -0.501 \\
\hline $\mathrm{C}\left(\mathrm{CH}_{3}\right)_{3}$ & 1.361 & -138.25 & -0.705 & 1.249 & -194.66 & -0.497 \\
\hline $\mathrm{Br}$ & 1.357 & -113.32 & -0.699 & 1.246 & -170.17 & -0.486 \\
\hline $\mathrm{Cl}$ & 1.357 & -112.88 & -0.700 & 1.246 & -169.23 & -0.487 \\
\hline Ophenyl & 1.360 & & -0.703 & 1.247 & & -0.513 \\
\hline $\mathrm{CH}_{3}$ & 1.361 & -138.19 & -0.705 & 1.248 & -191.80 & -0.498 \\
\hline $\mathrm{F}$ & 1.361 & -123.27 & -0.704 & 1.248 & -175.53 & -0.492 \\
\hline $\mathrm{OH}$ & 1.364 & -137.19 & -0.709 & 1.247 & -197.81 & -0.513 \\
\hline $\mathrm{OCH}_{2} \mathrm{CH}_{3}$ & 1.364 & -140.90 & -0.709 & 1.248 & -207.11 & -0.517 \\
\hline $\mathrm{OCH}_{3}$ & 1.364 & -140.06 & -0.708 & 1.248 & -203.92 & -0.515 \\
\hline $\mathrm{NH}_{2}$ & 1.366 & & -0.711 & 1.247 & -221.16 & -0.536 \\
\hline $\mathrm{N}\left(\mathrm{CH}_{3}\right)_{2}$ & 1.367 & -155.28 & -0.712 & 1.247 & -231.90 & -0.543 \\
\hline
\end{tabular}

of para-substituted phenols and their corresponding radicals. The decrease in $\mathrm{C}-\mathrm{O}$ bond length for neutral phenol molecules from bottom to top of the table indicates the increase of $\pi$-delocalization with the strength of the EW group, as was seen for anilines. Furthermore, the $\mathrm{C}-\mathrm{O}$ bond length in neutral phenolic compounds is correlated with $\mathrm{RB}_{\mathrm{C}-\mathrm{O}}$ (S5). The $\mathrm{C}-\mathrm{O}$ bond in phenoxyl radicals, on the other hand, is almost constant at $1.247 \AA$ for all para-substituents. Moreover, this $\mathrm{C}-\mathrm{O}$ bond length in phenoxyl radicals is closer to $\mathrm{C}=\mathrm{O}$ bond distance in aldehydes (1.210 $\mathrm{A})$ than $\mathrm{C}-\mathrm{O}$ bond distance in phenols (1.360 $\AA$ ), as calculated with $\omega B$ 97X-D level of theory.

Spin density map for unsubstituted phenoxyl and para- $\mathrm{N}, \mathrm{N}$-dimethylamino phenoxyl radicals is shown in Fig. 6. As with anilinyl radicals, the unpaired electron is located mainly in the vicinity of $\dot{O}$ atom and the ortho and para positions. However, spin density is less intense on $\dot{O}$ than the ring positions. In the presence of strong electron-donor $\mathrm{N}\left(\mathrm{CH}_{3}\right)_{2}$, the spin intensity is less than that of unsubstituted phenoxyl radical. Only in the case of para-amino phenoxyl radical, a tiny fraction of 


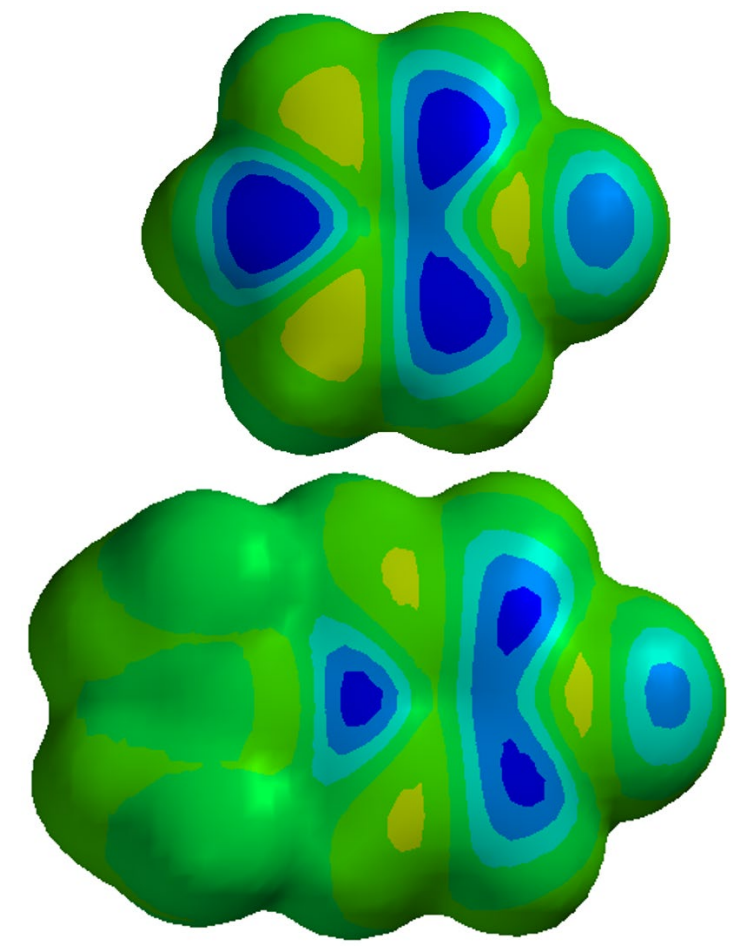

Fig 6. Spin density map for unsubstituted phenoxyl (top) and para- $N, N$-dimethylamino phenoxyl (bottom) radicals

spin density was observed on the amino $\dot{\mathrm{N}}$ as well. The spin density maps of phenoxyl radicals with EW groups and borderline substituents are similar to that of unsubstituted phenoxyl radicals.

An examination of Table 5 further reveals an increase in negative natural charge on hydroxyl $O$ and the minimum electrostatic potential around $\mathrm{O}$ with the rise in the electron-donating ability of para-substituent in both neutral phenols and their radicals.

The role of ED groups in increasing the negative charge on Ó of phenoxyl radical has also been reported in the literature [33]. Therefore, in para-substituted anilines, the electronic density around the vicinity of $\mathrm{O}$ and $\mathrm{O}$ atoms is enhanced with the increase of the electrondonating power of the para-substituent. Moreover, the change in electronic density around hydroxyl $\mathrm{O}$ in neutral molecules is linearly proportional to that on $\dot{O}$ in phenoxyl (Figs. S6 and S7).

\section{Origin of Substituent Effect}

The RB was confirmed as a measure of stability in neutral systems similar to the documented literature [35-
37] regarding the extent of resonance and inductive effects. Here it is demonstrated clearly that $\mathrm{N}-\mathrm{H}$ and $\mathrm{O}-$ $\mathrm{H}$ BDEs/BDEts correlate well with $\mathrm{RB}$ in neutral parasubstituted phenolic and anilinic compounds. Moreover, geometrical and spectroscopic parameters that are also expected to change with resonance or inductive stabilization power of the para-substituents in the neutral molecules are also shown to correlate well with $\mathrm{BDE} / \mathrm{BDEt}$ and $\mathrm{RB}$. On the contrary, such geometric parameters are independent of the nature of the para-substituent in the formed phenoxyl and anilinyl radicals.

It is shown that there is a noticeable interaction of $\dot{\mathrm{NH}}$ and $\dot{\mathrm{O}}$ unpaired electrons with phenyl carbons regardless of the nature of the para-substituent in both para-substituted anilinyl and phenoxyl radicals. There is a significant increase in the double-bond character of the radical $\mathrm{C}-\dot{\mathrm{N}}$ and $\mathrm{C}-\dot{\mathrm{O}}$ bonds with all substituents. Since the geometry around the heteroatoms is fixed and not affected by substituent identity, it can be suggested that there is resonance stabilization of the unpaired electron with the phenyl ring but not with the para-substituent for both types of radicals and with all substituents. This resonance phenomenon is further validated by the spin density maps for the phenoxyl and anilinyl radicals, where no spin fraction is observed at the parasubstituent region and by $\mathrm{N}-\mathrm{H}$ stretching vibrations that are almost constant with the variously parasubstituents. By comparing the spin density distribution for anilinyl and phenoxyl radicals in Fig. 3 and 6, it can be concluded that $\mathrm{O}$ group is a better electron acceptor than the $\dot{\mathrm{N}} \mathrm{H}$ group. This fact was observed before and attributed to shorter $\mathrm{C}-\mathrm{O}$ bonds than $\mathrm{N}-\mathrm{H}$ bonds [29].

It is observable from the spin density maps that there is a decrease in spin density in the phenyl ring in the presence of electron-donors in anilinyl and phenoxyl radicals. Furthermore, spin delocalization in radicals in strong para-ED groups is further stabilized by increasing electronic density around $\dot{\mathrm{NH}}$ and.O moieties induced by these groups. Therefore, it can be assumed that there is some extra stabilization of radicals in the case of strong electron-donors, and this is expected to decrease the $\mathrm{BDEs} / \mathrm{BDEts}$ further. It was suggested before that $\mathrm{ED}$ 
groups in para-substituted anilines and phenols stabilize the formed radicals by delocalizing the unpaired electron [31], especially $\mathrm{N}\left(\mathrm{CH}_{3}\right)_{2}$ and $\mathrm{NH}_{2}$ groups [22]. In this work, the data for $\mathrm{N}\left(\mathrm{CH}_{3}\right)_{2}$ and $\mathrm{NH}_{2}$ groups are outliners toward lower $\mathrm{N}-\mathrm{H}$ and $\mathrm{O}-\mathrm{H}$ BDEs/BDEts in Fig. 1 and 5. However, the stabilization of radicals by ED parasubstituents plays a small effect in determining BDE/BDEt for anilines and phenols, especially since the shift of electronic density toward the heteroatoms of the radicals is proportional to the increase in electronic density they induce on neutral molecules. Also, spin delocalization is observed by all substituents in anilinyl and phenoxyl radicals.

This work shows that the effect of para-EWsubstituents on BDE/BDEt due to $\pi$-delocalization and inductive effects in the parent neutral molecules is significantly more important than their effect on the corresponding radicals. Furthermore, it is also confirmed by the similarity of spin density distribution between unsubstituted and para-EW-substituted anilines and phenols. Therefore, the unpaired electron delocalization in radicals with EW groups [33] does not seem to add extra stabilization compared to unsubstituted compounds. The decrease in electronic density opposes the effect of spin delocalization observed with the electron-acceptors.

From above, it can be suggested that $\mathrm{N}-\mathrm{H}$ and $\mathrm{O}-\mathrm{H}$ $\mathrm{BDEs} / \mathrm{BDEts}$ are determined primarily by the stabilization/destabilization of the parent neutral molecules than the formed radicals. This study used several structural, atomic, and molecular properties to decide on the effect of substituent on both neutral molecules and radicals. In addition, the study provides, for the first time, the correlation between BDE/BDEt and several geometrical, atomic, molecular, and spectroscopic descriptors with RB to confirm that stabilization/ destabilization of the parent molecules is the main factor in the evaluation of BDE/BDEt values.

The calculated $\mathrm{RB}_{\mathrm{C}-\mathrm{O}}$ in para-substituted phenols fall in a narrower range than those of para-substituted anilines for all substituents, and the content of $\mathrm{RB}_{\mathrm{C}-\mathrm{O}}$ values of phenolic compounds is only $2.26 \mathrm{kcal} / \mathrm{mol}$ compared to $4.98 \mathrm{kcal} / \mathrm{mol}$ of anilinic compounds. This data trend can be understood by noting that the change of $\mathrm{C}-\mathrm{N}$ bond length is almost double that of $\mathrm{C}-\mathrm{O}$ length compared to unsubstituted aniline and phenol, as shown in Tables 2 and 5. Also, the N-H BDEs/BDEts of anilines are more significant than the $\mathrm{O}-\mathrm{H}$ BDEs/BDEts of phenols because $\mathrm{NH}_{2}$ is a more robust $\pi$-electron donor than $\mathrm{OH}[29]$ in the neutral compounds.

From the present study results, it can also be concluded that both BDE and BDEt are valid for investigating the substituent effect in molecules similar to those studied in this work. However, calculated BDEts reproduce better the experimental N-H BDE. Finally, The exchange-correlation functional $\omega \mathrm{B} 97 \mathrm{X}-\mathrm{D}$ used in this work produces the measured BDE for both anilinic and phenolic compounds.

\section{- CONCLUSION}

The calculated $\mathrm{N}-\mathrm{H}$ and $\mathrm{O}-\mathrm{H}$ BDE/BDEt of parasubstituted anilines and phenols showed a strong correlation with $\mathrm{RB}$ around phenyl- $\mathrm{NH}_{2}$ and phenyl$\mathrm{OH}$ bonds of neutral anilinic and phenolic compounds. The para-EW groups were found to cause an increase in $\mathrm{N}-\mathrm{H}$ and $\mathrm{OH}$ BDEs/BDEts, while results revealed the opposite behavior for ED groups. Geometric, atomic, and molecular properties of the $\mathrm{NH}_{2}$ and $\mathrm{OH}$ groups correlated well with the $\mathrm{RB}$ and $\mathrm{BDE} / \mathrm{BDEt}$. In the case of radicals, the geometrical changes around $\dot{\mathrm{N}} \mathrm{H}$ and $\dot{O}$ moieties remained constant for all substituents. Spin delocalization that involves the heteroatoms and the phenyl ring was observed for all substituents in anilinyl and phenoxyl radicals. The increase in electronic density around $\dot{\mathrm{NH}}$ and $\dot{\mathrm{O}}$ moieties of the radicals was proportional to that in neutral molecules. Based on the obtained results, $\mathrm{N}-\mathrm{H}$ and $\mathrm{OH}$ BDE/BDEt are determined mainly by stabilization/destabilization of neutral molecules except in the case of strong ED groups where minimal reduction in values of $\mathrm{N}-\mathrm{H}$ and $\mathrm{O}-\mathrm{H}$ BDE/BDEt occurs.

\section{- REFERENCES}

[1] Horton, W., Peerannawar, S., Török, B., and Török, M., 2019, Theoretical and experimental analysis of the antioxidant features of substituted phenol and 
aniline model compounds, Struct. Chem., 30 (1), 2335.

[2] Biela, M., Pelikánová, B., and Michalík, M., 2019, Antioxidant action of phenols: Revisiting theoretical calculations of their thermodynamics, Acta Chim. Slovaca, 12 (2), 212-217.

[3] Poliak, P., Vagánek, A., Lukeš, V., and Klein, E., 2015, Substitution and torsional effects on the energetics of homolytic N-H bond cleavage in diphenylamines, Polym. Degrad. Stab., 114, 37-44.

[4] Ali, H.M., Abo-Shady, A., Eldeen, H.A.S., Soror, H.A., Shousha, W.G., Abdel-Barry, O.A., and Saleh, A.M., 2013, Structural features, kinetics and SAR study of radical scavenging and antioxidant activities of phenolic and anilinic compounds, Chem. Cent. J., 7 (1), 53.

[5] Parcheta, M., Świsłocka, R., Orzechowska, S., Akimowicz, M., Choińska, R., and Lewandowski, W., 2021, Recent developments in effective antioxidants: The structure and antioxidant properties, Materials, 14 (8), 1984.

[6] Khalil, I., Yehye, W.A., Etxeberria, A.E., Alhadi, A.A., Dezfooli, S.M., Julkapli, N.B.M., Basirun, W.J., and Seyfoddin, A., 2020, Nanoantioxidants: Recent trends in antioxidant delivery applications, Antioxidants, 9 (1), 24.

[7] Maraveas, C., Bayer, I.S., and Bartzanas, T., 2021, Recent advances in antioxidant polymers: From sustainable and natural monomers to synthesis and applications, Polymers, 13 (15), 2465.

[8] Vo, Q.V., Nam, P.C., Thong, N.M., Trung, N.T., Phan, C.T.D. and Mechler, A., 2019, Antioxidant motifs in flavonoids: $\mathrm{O}-\mathrm{H}$ versus $\mathrm{C}-\mathrm{H}$ bond dissociation, ACS Omega, 4 (5), 8935-8942.

[9] Thong, N.M., Duong, T., Pham, L.T., and Nam, P.C., 2014, Theoretical investigation on the bond dissociation enthalpies of phenolic compounds extracted from Artocarpus altilis using ONIOM (ROB3LYP/6-311++G(2df,2p):PM6) method, Chem. Phys. Lett., 613, 139-145.

[10] Alisi, I.O., Uzairu, A., and Abechi, S.E., 2020, Free radical scavenging mechanism of 1,3,4-oxadiazole derivatives: Thermodynamics of $\mathrm{O}-\mathrm{H}$ and $\mathrm{N}-\mathrm{H}$ bond cleavage, Heliyon, 6 (3), e03683.

[11] Boli, L.S.P., Rusydi, F., Khoirunisa, V., Puspitasari, I., Rachmawati, H., and Dipojono, H.K., 2021, O-H and $\mathrm{C}-\mathrm{H}$ bond dissociations in non-phenyl and phenyl groups: A DFT study with dispersion and long-range corrections, Theor. Chem. Acc., 140 (7), 94.

[12] Beya, M.M., Netzel, M.E., Sultanbawa, Y., Smyth, H., and Hoffman, L.C., 2021, Plant-based phenolic molecules as natural preservatives in comminuted meats: A review, Antioxidants, 10 (2), 263.

[13] Ali, H.M., and Ali, I.H., 2015, QSAR and mechanisms of radical scavenging activity of phenolic and anilinic compounds using structural, electronic, kinetic, and thermodynamic parameters, Med. Chem. Res., 24 (3), 987-998.

[14] Sharopov, F.S., Wink, M., and Setzer, W.N., 2015, Radical scavenging and antioxidant activities of essential oil components-An experimental and computational investigation, Nat. Prod. Commun., 10 (1), 153-156.

[15] Stepanić, V., Trošelj, K.G., Lučić, B., Marković, Z., and Amić, D., 2013, Bond dissociation free energy as a general parameter for flavonoid radical scavenging activity, Food Chem., 141 (2), 15621570.

[16] Saqib, M., Mahmood, A., Akram, R., Khalid, B., Afzal, S., and Kamal, G.M., 2015, Density functional theory for exploring the structural characteristics and their effects on the antioxidant properties, $J$. Pharm. Appl. Chem., 1 (2), 65-71.

[17] Bendary, E., Francis, R.R., Ali, H.M.G., Sarwat, M.I., and El Hady, S., 2013, Antioxidant and structureactivity relationships (SARs) of some phenolic and anilines compounds, Ann. Agric. Sci., 58 (2), 173181.

[18] John, P.C.S., Guan, Y., Kim, Y., Kim, S., and Paton, R.S., 2020, Prediction of organic homolytic bond dissociation enthalpies at near chemical accuracy with sub-second computational cost, Nat. Commun., 11 (1), 2328. 
[19] Galano, A., Muñoz-Rugeles, L., Alvarez-Idaboy, J.R., Bao, J.L., and Truhlar, D.G., 2016, Hydrogen abstraction reactions from phenolic compounds by peroxyl radicals: Multireference character and density functional theory rate constants, J. Phys. Chem. A, 120 (27), 4634-4642.

[20] Liu, M., Zhang, Z., Chen, B., Meng, Q., Zhang, P., Song, J., and Han, B., 2020, Synthesis of thioethers, arenes and arylated benzoxazoles by transformation of the C (aryl)-C bond of aryl alcohols, Chem. Sci., 11 (29), 7634-7640.

[21] Lai, W., Li, C., Chen, H., and Shaik, S., 2012, Hydrogen-abstraction reactivity patterns from A to Y: The valence bond way, Angew. Chem. Int. Ed., 51 (23), 5556-5578.

[22] Garrett, G.E., Pratt, D.A., and Parent, J.S., 2020, Hydrogen atom abstraction from polyolefins: Experimental and computational studies of model systems, Macromolecules, 53 (8), 2793-2800.

[23] Aliaga, C., Almodovar, I., and Rezende, M.C., 2015, A single theoretical descriptor for the bonddissociation energy of substituted phenols, J. Mol. Model., 21 (1), 12.

[24] Khursan, S.L., 2016, Homodesmotic method of determining the $\mathrm{O}-\mathrm{H}$ bond dissociation energies in phenols, Kinet. Catal., 57 (2), 159-169.

[25] Denisov, E.T., and Denisova, T.G., 2015, Dissociation energies of $\mathrm{NH}$ bonds in aromatic amines, Pet. Chem., 55 (2), 85-103.

[26] Vagánek, A., Rimarčík, J., Ilčin, M., Škorňa, P., Lukeš, V., and Klein, E., 2013, Homolytic N-H bond cleavage in anilines: Energetics and substituent effect, Comput. Theor. Chem., 1014, 60-67.

[27] Klein, E., and Lukeš, V., 2006, Study of gas-phase O$\mathrm{H}$ bond dissociation enthalpies and ionization potentials of substituted phenols-applicability of $a b$ initio and DFT/B3LYP methods, Chem. Phys., 330 (3), 515-525.

[28] Li, Z., and Cheng, J.P., 2003, A detailed investigation of subsitituent effects on $\mathrm{N}-\mathrm{H}$ bond enthalpies in aniline derivatives and on the stability of corresponding N-centered radicals, J. Org. Chem., 68 (19), 7350-7360.
[29] Pratt, D.A., DiLabio, G.A., Valgimigli, L., Pedulli, G.F., and Ingold, K.U., 2002, Substituent effects on the bond dissociation enthalpies of aromatic amines, J. Am. Chem. Soc., 124 (37), 11085-11092.

[30] Jonsson, M., Lind, J., Merényi, G., and Eriksen, T.E., 1995, N-H bond dissociation energies, reduction potentials and $\mathrm{pK}_{\mathrm{a}} \mathrm{s}$ of multisubstituted anilines and aniline radical cations, J. Chem. Soc., Perkin Trans., 2 (1), 61-65.

[31] Bordwell, F.G., Zhang, X.M., and Cheng, J.P., 1993, Bond dissociation energies of the nitrogenhydrogen bonds in anilines and in the corresponding radical anions. Equilibrium acidities of aniline radical cations, J. Org. Chem., 58 (23), 6410-6416.

[32] Song, K.S., Liu, L., and Guo, Q.X., 2003, Remote Substituent effects on $\mathrm{N}-\mathrm{X}\left(\mathrm{X}=\mathrm{H}, \mathrm{F}, \mathrm{Cl}, \mathrm{CH}_{3}, \mathrm{Li}\right)$ bond dissociation energies in para-substituted anilines, J. Org. Chem., 68 (2), 262-266.

[33] Wu, Y.D., and Lai, D.K.W., 1996, A density functional study of substituent effects on the $\mathrm{O}-\mathrm{H}$ and $\mathrm{O}-\mathrm{CH}_{3}$ bond dissociation energies in phenol and anisole, J. Org. Chem., 61 (22), 7904-7910.

[34] Zhang, H.Y., Sun, YM., and Chen, D.Z., 2001, O-H bond dissociation energies of phenolic compounds are determined by field/inductive effect or resonance effect? A DFT study and its implication, Quant. Struct.-Act. Relat., 20 (2), 148-152.

[35] Yateem, A.H., 2020, Rotational barrier and electronwithdrawing substituent effects: Theoretical study of $\pi$-conjugation in para-substituted anilines, Mediterr. J. Chem., 10 (4), 319-334.

[36] Yateem, A.H., 2020, Rotational barrier and quantification of electron-donating substituent effects: A computational study of para-substituted benzaldehydes, Croat. Chem. Acta, 93 (2), 85-96.

[37] Yateem, A.H., 2019, Rotational barrier and conjugation: Theoretical study of resonance stabilization of various substituents for the donors $\mathrm{NH}_{2}$ and $\mathrm{OCH}_{3}$ in substituted 1,3-butadienes, Indones. J. Chem., 19 (4), 1055-1065.

[38] Jacobsen, H., and Cavallo, L., 2017, "Directions for Use of Density Functional Theory: A Short 
Instruction Manual for Chemists" in Handbook of Computational Chemistry, Eds., Leszczynski, J., Kaczmarek-Kedziera, A., Puzyn, T., Papadopoulos, M.G., Reis H., Shukla M.K., Springer, Cham, 225267.

[39] Mardirossian, N., and Head-Gordon, M., 2017, Thirty years of density functional theory in computational chemistry: an overview and extensive assessment of 200 density functionals, Mol. Phys., 115 (19), 2315-2372.
[40] SPARTAN'14, 2014, Wavefunction, Irvine, CA, USA.

[41] Bruno, G., Macetti, G., Lo Presti, L., and Gatti, C., 2020, Spin density topology, Molecules, 25 (15), 3537.

[42] Grozav, A., Porumb, I.D., Găină, L.I., Filip, L., and Hanganu, D., 2017, Cytotoxicity and antioxidant potential of novel 2-(2-((1H-indol-5yl) methylene)hydrazinyl)-thiazole derivatives, Molecules, 22 (2), 260. 